\title{
Theory of Protostellar Objects
}

\author{
Frank H. Shu
}

Institute for Theoretical Physics, University of California, Santa Barbara, CA 93106, USA

\begin{abstract}
Many problems in the theory of star formation are amenable to a complementary attack in which the analytical approach is used to reduce the governing equations to a form amenable to efficient numerical solution. This strategy has proven very useful in helping to resolve several astrophysical puzzles which arise because the bulk of star formation today is observed to occur, with relatively low efficiency, in giant molecular cloud complexes. How does a cloud of $10^{5}-10^{6} M_{\odot}$ know how to form stars of mass $\sim 1 M_{\odot}$ ? How does the interstellar medium know, to one or two orders of magnitude, that roughly $\left(\hbar c / G m_{\mathrm{H}}^{2}\right)^{3 / 2}$ hydrogen atoms of mass $m_{\mathrm{H}}$ are needed to yield thermonuclear fusion in a self-gravitating ball of gas? Why have radio astronomers not detected unambiguous evidence for the collapse motions attendant to star formation? Why has a true protostar, the "holy grail" of infrared astronomy, been so hard to find? Why do young stellar objects almost universally exhibit powerful outflows? Why is the geometry for these outflows often bipolar? Why do T Tauri stars have such active chromospheres? In this review we suggest that these puzzles all have a related resolution, in the nature of how gravitational collapse is initiated and terminated in the slowly rotating cores of molecular clouds.
\end{abstract}

\section{Introduction}

Following the pioneering work of HAYASHI [1] and LARSON [2], it is commonly accepted (a) that the birth of a star involves gravitational collapse from an extended cloud of gas and dust, and (b) that a protostar builds up by accretion from the infalling material. This conventional picture presents a considerable challenge for the subject of radiative hydrodynamics because the collapse from interstellar dimensions to stellar ones involves a very large dynamical range, e.g., more than twenty orders of magnitude changes in density. The numerical difficulties associated with following the complete evolution of a protostellar object by pure finitedifference schemes, even in spherical symmetry, led to considerable controversy in the field [3-7]. When the effects of rotation and magnetic fields are included, the numerical problems are compounded [8-17], and to date no theorist has succeeded in rigorously producing stars from interstellar clouds with variations in more than one spatial dimension.

From another point of view, however, it is clear that the existence of large (or small) parameters may offer considerable scope for analytical techniques: simi- 
larity solutions [18-21], perturbation techniques [22-24], matched asymptotic expansions [25], etc. Since the finite-difference approach has been amply reviewed elsewhere [26-30], I shall concentrate here on the semi-analytical methods which can be used to reduce the governing equations to a form amenable to quick and accurate numerical solution. Numerical integrations are usually required at the last stage of the analysis if sufficient physical realism is retained to allow meaningful comparisons of the computed results with observational data. Thus, analytical and numerical techniques should be regarded in this field, as in many others, as being complementary rather than being competitive.

\section{The Mass Scale of Stars}

To begin a discussion of the process of star formation, we should first define what we mean by a (normal) star. The following definition would probably satisfy most astronomers and nuclear physicists: A normal star is a luminous ball of selfgravitating gas which possesses sufficient mass to enable thermonuclear fusion in its central regions. This immediately raises the interesting question: After mass loss, how heavy does an isolated star have to be for fusion reactions to provide a major source of energy? The answer given by stellar evolution theory (see [31] and refs. therein) is, to a level of accuracy sufficient for our purposes,

Deuterium burning only: $0.01 M_{\odot}<M_{*}<0.08 M_{\odot}$,

Hydrogen burning also: $0.08 M_{\odot}<M_{*}<0.4 M_{\odot}$,

Helium burning also: $0.4 M_{\odot}<M_{*}<1.4 M_{\odot}$,

Iron and beyond: $M_{*}>1.4 M_{\odot}$.

The value $1.4 M_{\odot}$ represents, of course, Chandrasekhar's limiting mass for a white dwarf: $0.78\left(\hbar c / G m_{p}^{2}\right)^{3 / 2} m_{p}$, where $m_{p}$ is the mass of the proton and the other symbols have their usual meanings. Our understanding of the mass ranges given above rests with the notion that all stars evolve as to try ultimately to produce a white dwarf at its core (for massive stars, this is the pre-supernova state). If this white dwarf has a mass which approaches Chandrasekhar's limiting mass, then it can force the pressures and temperatures in the part of the star just above it to almost arbitrarily high values - values sufficient, indeed, to produce all the chemical species in the periodic table up to iron (by charged particle reactions) and beyond (by neutron capture reactions). On the other hand, if the final whitedwarf core is less than Chandrasekhar's limit, then nuclear burning is terminated at an earlier stage.

The most common stars in our Galaxy and other giant galaxies seem to have masses of $0.5 M_{\odot}$ or less [32]; thus, within a factor of 3 or so, Chandrasekhar's limit seems to be a natural unit for the mass scale of stars. This central fact can be regarded as the fundamental issue which must be addressed by any viable theory of star formation: How does a galaxy of mass $10^{11} \mathrm{M}_{\odot}$ and size $10^{23} \mathrm{~cm}$ know how to form objects of mass $10^{0} M_{\odot}$ and size $10^{11} \mathrm{~cm}$ ? Most astronomers today would 
split this problem into two subproblems, i.e., they would draw a schematic flow diagram of the entire process as

$$
\text { Galaxy } \rightarrow \text { Giant Molecular Cloud Complexes } \rightarrow \text { Stars, }
$$

with giant molecular cloud complexes (GMCs) having intermediate masses ( $\left.10^{5.5} M_{\odot}\right)$ and sizes $\left(\sim 10^{20} \mathrm{~cm}\right)$ to galaxies and stars. I wish to discuss here only the second subproblem, namely, How does a GMC with mass much greater than that of any star know how to form gravitationally bound subunits only marginally capable of thermonuclear fusion, i.e., objects which contain $\sim\left(\hbar c / G m_{\mathrm{H}}^{2}\right)^{3 / 2}$ hy. drogen atoms of mass $m_{\mathrm{H}}$ ?

Until a few years ago, the prevailing attitude on this issue was that the mass scale of stars is largely a coincidence, a consequence of the fragmentation of large aggregates of gaseous matter into smaller pieces - in its grandest manifestation, from galactic scales to molecular-cloud scales to stellar scales. In this view (e.g., expressed in refs. [33-38]), the endpoint of the fragmentation process, after cascading through many orders of magnitude, resulted from physical processes which were completely unrelated to the particular combination of fundamental physical constants that enter in Chandrasekhar's limit. If this were true, then one would be left with the puzzle why systems with as disparate physical conditions and starformation efficiencies as elliptical galaxies, spiral galaxies (with and without "grand designs"), irregular galaxies, globular clusters, open clusters, $\mathrm{T}$ associations, etc., have all nevertheless managed to produce mostly stars of a Chandrasekhar mass or less. Except perhaps for a hypothetical Population III which produced the initial heavy-element content of Population II, low-mass stars are the typical outcome of the process of star formation; high-mass stars are much more spectacular, but they are atypical.

In my opinion [39], the mass unit $\left(\hbar c / G m_{\mathrm{H}}^{2}\right)^{3 / 2} m_{\mathrm{H}}$ is fundamental, not only to the structure of stars, but also to their formation; however, it is not the interstellar medium which knows this number, it is stars themselves. The key, I believe, lies in how the star formation process (collapse followed by accretion) is terminated, and the current observational evidence suggests stellar winds. Indeed, one of the most remarkable developments in this field has been the observational discovery that probably all young stellar objects undergo a phase of intense mass loss, and that the resulting outflows often take both jetlike and bipolar forms [40-43]. We are therefore left with the problem of explaining why such outflows should be a natural consequence of the (star formation) processes which preceeded it, why they usually occur after a star has accumulated on the order of a Chandrasekhar's mass or less of material, and why the winds should be channeled anisotropically when they (first) develop. In what follows, I shall outline a picture, via a series of simple idealized calculations, of how the whole process may work in the simplest context when a molecular cloud (core) is left free to evolve in isolation from its environment. I do not claim that the picture is complete, for there are gaps where there do not exist even simplified calculations, and the theory as developed does not account for the formation of massive stars, binary stars, bound clusters, and 
other important phenomena. The hope is that if we can make progress on the simplest situation, we will be able to see what conditions need to be violated to produce other outcomes.

\section{The Mechanical Support of Molecular Clouds}

Radio astronomical maps [44,45] of relatively nearby GMCs show them to have volume-averaged densities $\sim 30 \mathrm{H}_{2} \mathrm{~cm}^{-3}$ and temperatures $\sim 10 \mathrm{~K}$. Under these conditions, a $10^{5} M_{\odot}$ GMC contains about $10^{4}$ Jeans masses, which implies that it cannot be supported against its self-gravitation by thermal pressure. On the other hand, the free-fall time for a GMC is only $\sim 6 \times 10^{6} \mathrm{yr}$, and if it has a star formation efficiency typical of GMCs observed in the inner Galaxy, it converts only $1 \%$ of its mass into stars over such a time span. This combination suggests that GMCs cannot be anywhere near freely falling, and they must be supported mechanically by some means [46]. At various times, rotation [47], turbulence [48.49], and magnetic fields $[50,51]$ have been invoked as prime possibilities.

Observed in the rotational transitions of $\mathrm{CO}, \mathrm{GMCs}$ are typically clumped on scales of a few pc, and the clumps of $\sim 10^{3} M_{\odot}$ each have average $\mathrm{H}_{2}$ densities of a few hundred $\mathrm{cm}^{-3}$. There is probably nothing characteristic about clump densities of a few hundred $\mathrm{cm}^{-3}$, they are simply the minimum values needed to excite $\mathrm{CO}$. Molecules which are harder to excite show clumps to have multiple cores with densities exceeding $10^{4}-10^{6} \mathrm{H}_{2} \mathrm{~cm}^{-3}$, and a hypothetical tracer sensitive to even lower densities would probably show "envelopes" that extend to the "background" level, $30 \mathrm{H}_{2} \mathrm{~cm}^{-3}$. A hint of the latter behavior exists in the knowledge that molecular clouds are often, perhaps always, surrounded by atomic hydrogen envelopes of lower density. In any case, the $\mathrm{CO}$ clumps are observed to move relative to one another at velocities of a few $\mathrm{km} \mathrm{s}^{-1}$, which allows the interpretation that their spatial distribution within a complex may be maintained by random motions, although magnetic support is another possibility. (Rotation is generally small on the scale of a complex as a whole [52].) Virial theorem arguments (or dimensional ones) give the following formula for the critical mass $M_{\mathrm{cr}}$ of (partially ionized) gas that can be supported by a magnetic flux $\Phi$ threading through it [53]:

$$
M_{\mathrm{cr}}=\alpha G^{-1 / 2} \Phi
$$

where $\alpha$ is a numerical coefficient of order unity. MOUSCHOVIAS and SPITZER [54] give $\alpha \approx 0.15$ to summarize the results of exact numerical calculations. If field of average strength $B=30 \mu \mathrm{G}$ threads through a cloud of radius $R, M_{\mathrm{cr}} \sim 10^{3} M_{\odot}$ if $R \sim 2 \mathrm{pc}$, whereas $M_{\mathrm{cr}} \sim 10^{5} M_{\odot}$ if $R \sim 20 \mathrm{pc}$. Thus, field strengths of the order of $30 \mu \mathrm{G}$ or more could go a long way to explaining the mechanical support of both individual molecular cloud clumps and giant complexes of molecular clouds. It is therefore significant that Zeeman splitting measurements of regions with molecular cloud densities yield values for $B$ which are in the general range of $10^{1}-10^{2} \mu \mathrm{G}$ [55].

Of course, static magnetic fields can exert no force in the direction parallel 
to their mean direction, and turbulence remains a viable candidate for this support. However, maps of the interstellar polarization produced in nearby dark clouds (which resemble the individual clumps inside more distant GMCs) show a high degree of correlation for the direction of the polarization vector over the dimensions of the entire cloud [56], implying that turbulence in these objects, at least, cannot be so strong as to completely tangle the magnetic field. This suggests that turbulence is, at best, competitive with but does dominate magnetic fields in the support of molecular cloud clumps. Indeed, since super-alfvenic or super-magnetosonic turbulence probably decays fairly quickly, it is tempting to speculate that the turbulence in molecular clouds (probably ultimately driven by stellar winds) is more wavelike than eddylike [57], oscillating about a mean field direction which is well defined, and that magnetic shocks usually keep the turbulence down to sub-alfvenic levels. Taken to an extreme, even the clumps of a molecular cloud complex might move sub-alfvenically with respect to each other, sending ahead gently rippling waves in the interclump medium to cushion impacts. Estimates of the rates of "turbulent" energy dissipation based on the assumption of sudden clump collisions may therefore require substantial modification.

For clouds which start with masses $M_{\mathrm{cl}}$ less than $M_{\mathrm{cr}}$, equation (1) has an important consequence which makes the problem of star formation in the presence of magnetic fields completely different from the problem in its absence. As MESTEL has stressed for many years [58], without a reduction of the flux to mass ratio (e.g., by a decrease in $\Phi$ or an increase in $M_{\mathrm{cl}}$ ) or a reduction of the coefficient $\alpha$ (e.g., by a geometric rearrangement of the material), no sufficiently magnetic cloud can ever be induced to collapse gravitationally, nor can any subpiece of it ever fragment out of the background. In particular, with flux freezing, external compression of a fixed amount of matter, by any means whatsoever, does not help unless the change in surface pressure or internal temperature or boundary shape is so great as to change $\alpha$ very appreciably from its unperturbed value. Even colliding two identical clouds head-on along the general direction of the magnetic lines of force will induce collapse only if the original clouds were already within a factor of 2 of gravitational instability. Thus, if $M_{\mathrm{cl}}<M_{\mathrm{cr}}$, the most reasonable means of inducing star formation in magnetic clouds would seem to be to reduce the magnetic flux $\Phi$ under conditions of quasi-magnetostatic equilibrium. This situation may apply to the Taurus dark cloud whose dense cores are extremely cold and quiescent, with low-mass star formation taking place at a slow measured pace $[59,60]$. In the less common case when $M_{\mathrm{cl}}>M_{c r}$ (e.g., possibly in Rho Ophiucus $[61,62])$, relatively rapid clump contraction, leading to a massive cloud "core," may occur simultaneously with the formation of a (bound) cluster of multiple dense (sub)cores of the Taurus type and protostars. In any case, a large reduction of the flux to mass ratio $\Phi / M$ is necessary in star-forming regions since otherwise stars would form with magnetic flux which are many orders of magnitude larger than observed. Moreover, flux reduction by the process of ambipolar diffusion is only to be expected in molecular clouds which contain very little fractional ionization, and as we shall see, this process leads naturally to conditions 
conducive to the formation of low-mass stars.

\section{The Formation of Molecular Cloud Cores}

If magnetic fields do provide support for molecular cloud clumps (of masses $10^{3}$ $10^{4} M_{\odot}$ ), then it is inevitable that such clumps will develop subclumping, i.e., molecular cloud cores. The reason is that magnetic stresses can be exerted directly only on the charged component of the medium (to which the magnetic field is effectively frozen) while the material of a molecular cloud is mostly neutral. If we ignore the role of charged grains, the magnetic field provides support for the cloud only because the neutrals slip relative to the ions (and electrons) at a relative velocity $\mathbf{u}-\mathbf{u}_{i}$, and this slip (ambipolar diffusion [63-67]) generates a frictional force per unit volume equal to

$$
\mathbf{f}_{\mathrm{drag}}=-\gamma \rho \rho_{i}\left(\mathbf{u}-\mathbf{u}_{i}\right)
$$

where $\gamma$ is the drag coefficient associated with momentum exchange in ion-neutral collisions [68]. Thus, magnetic support of self-gravitating matter of low fractional ionization necessarily involves slip of the neutral component of that matter relative to the field and the gradual concentration of the neutrals into subcondensations where the magnetic field plays increasingly less of a part in the total support.

A simple idealized problem illustrates the physical principle. Consider the magnetic support of a plane-parallel slab of gas of neutral volume density $\rho(z)$ which extends infinitely in $z$, and where $\mathbf{B}(z)$ is perpendicular to the $z$ axis. We ignore possible instabilities in the horizontal directions and suppose that the evolution. time is long in comparison with the time scales for heating and cooling so that we may adopt an isothermal equation of state for the gas. We also suppose the evolutionary time is long in comparison with the time scales for ionization (by cosmic rays) and recombination (by two-body processes) so that the ion density. $\rho_{i}$ is given by a condition of ionization equilibrium:

$$
\rho_{i}=C \rho^{1 / 2}
$$

where the coefficient $C$ is a constant. If $\rho_{i} \ll \rho$, it can be shown that the time evolution of the magnetic flux to neutral mass ratio, $B / \rho$ for our one-dimensional problem, satisfies a nonlinear diffusion equation [69]:

$$
\frac{\partial}{\partial t}\left(\frac{B}{\rho}\right)=\frac{1}{\gamma} \frac{\partial}{\partial \sigma}\left(\frac{B^{2}}{4 \pi \rho_{i}} \frac{\partial B}{\partial \sigma}\right),
$$

in which we recognize the quantity $B^{2} / 4 \pi \rho_{i}$ as the square of the Alfven velocity in the ionized component alone, and where $\sigma$ is the surface density of neutrals between $z=0$ and $z$ :

$$
\sigma \equiv \int_{0}^{z} \rho\left(z^{\prime}, t\right) d z^{\prime}
$$


When the evolution time is also long in comparison with the free-fall time scale, the above set of equations is closed by the condition of magnetostatic balance:

$$
\frac{B^{2}}{8 \pi}+a^{2} \rho=2 \pi G\left(\sigma_{\infty}^{2}-\sigma^{2}\right)
$$

where $a$ is the isothermal speed of sound in the neutral gas and $\sigma_{\infty}$ is (half) its total mass column density.

To equation (4), we apply the boundary conditions:

$$
\begin{aligned}
& \frac{\partial B}{\partial \sigma}=0 \quad \text { at } \quad \sigma=0 \quad(z=0), \\
& B=0 \quad \text { at } \quad \sigma=\sigma_{\infty} \quad(z=\infty),
\end{aligned}
$$

and solve it subject to some reasonable initial conditions, such as magnetostatic equilibrium with a characteristic ratio $\alpha_{0}$ of magnetic pressure to gas pressure. The solutions, however, all have the interesting behavior that as $t \rightarrow \infty$, they approach unique asymptotic forms:

$$
\sigma \rightarrow \sigma_{\infty} \tanh \left(z / z_{0}\right), \quad \rho \rightarrow \frac{\sigma_{\infty}}{z_{0}} \operatorname{sech}^{2}\left(z / z_{0}\right), \quad B \rightarrow 4 \pi G^{1 / 2} \sigma_{\infty} \frac{Z\left(\sigma / \sigma_{\infty}\right)}{\left[2\left(\tau-\tau_{0}\right)\right]^{1 / 2}}
$$

where $z_{0} \equiv a^{2} / 2 \pi G \sigma_{\infty}, Z\left(\sigma / \sigma_{\infty}\right)$ is a unique function of its argument, $\tau$ is a dimensionless time variable,

$$
t \equiv\left[\frac{\gamma C}{2(2 \pi G)^{1 / 2}}\right]\left(\frac{z_{0}}{a}\right) \tau
$$

and $\tau_{0}$ is a (intermediate asymptotic) time constant which is the only quantity in the expressions that depends on the initial conditions (roughly $\tau_{0} \propto \alpha_{0}^{1 / 2}$ ).

The physical interpretation of the result (8) is straightforward. As $t \rightarrow \infty$, the magnetic field wants to decay to its background value (here, 0 ), and the gas wants to acquire the configuration that applies in the absence of a magnetic field, i.e., support against self-gravity by thermal pressure alone. With a large initial ratio of magnetic to thermal pressure, $\alpha_{0}$, the outcome is the production of a small dense core in the background of a more extended envelope (see fig. $2 \mathrm{c}$ of [69]). Values of interest to present-day molecular clumps would correspond to $\alpha_{0} \sim 10^{2}$ if the gas pressure were all thermal, but values closer to unity might apply if a "turbulent" contribution to $\rho a^{2}$ were considered. The proper inclusion of turbulence is a delicate issue since its presence may enhance ambipolar diffusion initially, but as the Alfven velocity drops, the turbulence (probably a spectrum of nonlinear waves) is also likely to decay. Thus, in a more realistic 3-D problem where the original cloud clump contains many Jeans masses, we may expect the clump to fragment quasi-statically into many "cores," each core asymptotically trying to reach a configuration where the field lines become almost uniform and straight (the 
background condition) and where the total velocity dispersions approach purely thermal values. This picture constitutes a very attractive explanation for the quiet molecular cloud cores which are observed in the Taurus dark cloud by MYERS and BENSON [60]. However, stable asymptotic support by thermal pressure alone in quasi-spherical symmetry is impossible if the potential reservoir of matter is very large in comparison with a Jeans mass in the initial state; thus, 3-D isothermal cores must ultimately undergo gravitational collapse when they become sufficiently centrally condensed. This has apparently happened in the Taurus region to roughly half of Myers and Benson's cores, which are known to be the sites of T Tauri star formation. The theoretical elucidation of how and when quasi-static evolution is tranformed into dynamical collapse is an ongoing project of LIZANO and SHU [70].

A rough estimate for the time required for molecular cloud core formation is provided by the 1-D calculations:

$$
\tau_{0}\left[\frac{\gamma C}{2(2 \pi G)^{1 / 2}}\right]\left(\frac{z_{0}}{a}\right),
$$

where we recognize the factor $z_{0} / a$ as the sound travel time across the scale height of the core in the final state $\left(\sim 5 \times 10^{5} \mathrm{y}\right.$ if $z_{0} \sim 0.1 \mathrm{pc}$ and $\left.a \sim 0.2 \mathrm{~km} \mathrm{~s}^{-1}\right)$. Since the latter is the characteristic dynamical timescale of the system, we see that ambipolar diffusion can be considered a slow evolutionary process only to the extent that the coefficient,

$$
\alpha_{0}^{1 / 2}\left[\frac{\gamma C}{2(2 \pi G)^{1 / 2}}\right]
$$

is a pure number large compared to unity. For the conditions which apply typically in present-day molecular clouds, this number is large in comparison with unity, of the order of 10 or 100 , and this probably explains why star formation is generally an inefficient process in present-day molecular clouds. On the time scale of the dynamical collapse of unstable molecular cloud cores (the sound travel time across the core), star formation is a badly synchronized affair in molecular cloud clumps because the time it takes to form the many individual cores takes 10-100 times longer. This means that newly-formed T Tauri stars have ample time to turn on their winds and disrupt the incipient condensation of neighboring cores, making star formation in $\mathrm{T}$ associations a loosely aggregated affair. There is no guarantee, however, that the dimensionless combination represented by expression (10) is large compared to unity in all circumstances. Low fractional ionizations or low initial ratios of magnetic pressure to thermal pressure may well lead, in some circumstances, to relatively high-star formation efficiencies, especially if $M_{\mathrm{cl}}$ started out initially larger than $M_{\mathrm{cr}}$. Whether these are the crucial ingredients that allow the formation of bound clusters, open and globular, remain to be seen.

Slow slippage of magnetic field lines in the disk of the Galaxy provides two additionally welcome features to the theory of star formation in $\mathrm{T}$ associations. First, a slow initial phase of gravitational condensation almost guarantees magnetic 
braking [71-74] of the cores of molecular clouds to rotation rates characteristic of their envelopes before the cores undergo gravitational collapse. There will also be a tendency for angular velocity vector $\boldsymbol{\Omega}$ to align with the direction of the clump magnetic field because the perpendicular component of $\boldsymbol{\Omega}$ will brake more quickly than the parallel component. Thus, these molecular cloud cores will contain a rich supply of material with relatively low angular momentum from which to form binary stars and planetary systems. Second, the slow evolution of these objects toward a state of gravitational instability may give well-defined initial states for dynamical collapse calculations.

\section{The Gravitational Collapse of Well-Separated Molecular Cloud Cores}

In the absence of more detailed calculations, it is fruitful to speculate on what radial density profile is likely to result for a molecular cloud core as a consequence of the quasi-static process of ambipolar diffusion sketched in the last section. Nearly twenty years ago, BODENHEIMER and SWEIGART [75] pointed out that subsonic evolution of a non-rotating, non-magnetic cloud would always produce a $\rho \propto$ $r^{-2}$ density distribution. This is interesting because SHU [20] demonstrated later that the singular isothermal sphere,

$$
\rho=\frac{a^{2}}{2 \pi G r^{2}}
$$

has a self-similar collapse solution, whose form, apart from the integration of some simple ordinary differential equations, could be found analytically. Because the central density of the singular isothermal sphere is infinite, it might appear that starting conditions for the collapse problem based on this approximation are rather artificial [76]. However, it should be remembered that NAKANO [77] (see also $[78,79])$ found that when one includes grain coupling to ambipolar diffusion calculations, very high densities (compared to envelope values) can be reached in the central regions of a molecular cloud core before dynamical collapse is generated. As long as the power-law part of a real core solution spans several decades in density, except for initial transients, its gravitational collapse is likely to be well represented by the self-similar solution for the singular isothermal sphere (generalized, perhaps, to include the perturbational effects of the finite amounts of rotation and magnetic fields left by the process of diffusive core formation described earlier).

How well does equation (11) reproduce the observed features of real molecular cloud cores? The ammonia cores studied by MYERS [80] have line-widths corresponding to $a \approx 0.2 \mathrm{~km}^{-1}$ when they do not yet contain protostars. Equation (11) then predicts that over an observed radius $R \sim 3 \times 10^{17} \mathrm{~cm}$, covering the center of the core, the volume-averaged density $3 a^{2} / 2 \pi G R^{2}$ should be $\sim 10^{4}$ $\mathrm{H}_{2} \mathrm{~cm}^{-3}$, which is roughly the density required to collisionally excite ammonia [81]. In other words, given $T=10 \mathrm{~K}$, equation (11) predicts correctly the bulk properties of ammonia cores. Moreover, there are indications that observations in molecules sensitive to lower and higher densities yield core "sizes" (and core 
"masses") which are, respectively, larger and smaller than the ammonia values, consistent with $\rho$ being a monotonically decreasing function of increasing $r$ at least until $\mathrm{CO}$ excitation values are reached. It would be valuable to quantify these qualitative statements with precise observational determinations of the density profiles of molecular cloud cores.

In the absence of such determinations, it is important to note that if equation (11) is correct, there may be no such thing as a typical density or a typical mass for a molecular cloud core, because a power law has no typical scale. Stated in terms of the Jeans mass, we may say that equation (11) has no typical Jeans mass because it has no typical density, i.e., the singular isothermal sphere contains about one Jeans mass at every radius $r$. Except for the singularity at the center, this is the only possible hydrostatic resolution of an isothermal configuration which has access to a (virtually) unlimited supply of matter and which has every means of support other than thermal pressure slowly drained away from it. This also means that when such configurations go into dynamical collapse, the solutions do not automatically define a mass scale that we may associate with the formation of ordinary stars. What is well defined instead is a rate at which the central object is built up by accretion; in the self-similar solution for the singular isothermal sphere, this rate equals

$$
\dot{M}=0.975 a^{3} / G,
$$

which, for $a=0.2 \mathrm{~km} \mathrm{~s}^{-1}$, has the value $2 \times 10^{-6} M_{\odot} \mathrm{y}^{-1}$.

In the self-similar collapse solution, infall is initiated from "inside-out" by an expansion wave which propagates outward at the speed of sound into static material with the density distribution (11). The head of the expansion wave reaches radius $r_{h}=a t$ in time $t$; and supersonic inflow velocities are generated interior to $r_{0} \approx 0.4 a t$. Identifying $t=M / \dot{M}$ as the time required to build up the central star to a mass $M$ at the mass infall rate $(12)$, we easily calculate that $r_{0} \approx 7 \times 10^{1 \epsilon}$ $\mathrm{cm}$ for $M=0.5 M_{\odot}$ and $a=0.2 \mathrm{~km} \mathrm{~s}^{-1}$. Since infall velocities for $r<r_{\text {a }}$ scale approximately as $r^{-1 / 2}$, the detection of infall velocities exceeding, say, $0.5 \mathrm{~km}$ $\mathrm{s}^{-1}$, will require linear resolutions of $\sim 10^{16} \mathrm{~cm}$. This is only becoming possible now with a new generation of millimeter-wave telescopes, which may explain why radio astronomers have previously only found the much larger regions of high velocity outflows that are associated with a later phase of stellar evolution than the pure protostellar stage.

\section{The Structure and Evolution of Spherical Protostars}

The isothermal approximation begins to break down badly in low-mass protostars when the infalling dust and gas becomes optically thick to the emergent infrared radiation interior to a radius $r_{e}$ of about $10^{14} \mathrm{~cm}$. Fortunately, for $r$ less than the radius of the dust photosphere, $r_{e}$, and greater than the stellar radius, $R_{*} \sim 10^{11.5}$ $\mathrm{cm}$ (bounded by an accretion shock), the material falls nearly freely toward the mass at the center, so that the crossing time for either matter or radiation is short enough compared to the evolutionary time, and a condition of steady-state flow 
holds to a high degree of approximation. The inner region of complicated radiative hydrodynamics may thus be solved (using carefully chosen closure relations for the frequency-integrated moment equations of the radiation field) as a set of ordinary differential equations, alleviating greatly the computational demands of the problem [22]. The similarity solution discussed in the previous section provides outer boundary conditions for the inner problem, namely, inflow at free-fall speeds with mass infall rate (12).

The most important feature of the region $R_{*}<r<r_{e}$ is the opacity gap [24], which appears because the opacity drops by several orders of magnitude after dust grains are thermally and chemically destroyed but before the accretion shock (or the stellar [gas] photosphere) is crossed. The existence of an opacity gap is virtually foreordained by dust destruction temperatures $T_{d}$ being one to two thousand degrees lower than Hayashi's limiting temperature $T_{H}$, the lowest temperature accessible to gaseous photospheres [82]. The importance of the opacity gap is that it guarantees that gravitational binding energy liberated in the radiative shock at the stellar surface will eventually leave the configuration entirely (rather than be swept back into the star by the infalling matter). It also leads to an interesting problem in radiative transfer theory in the neighborhood of the dust destruction front, where an outwardly directed stellar radiation field is exponentially attenuated and thermalized to become the diffuse radiation field characteristic of the dust envelope. To handle this problem by the moment equation method, we found it useful to adopt the closure relation:

$$
\varepsilon=3 P-\frac{2}{c} \mu_{g} \varsubsetneqq,
$$

where $\mathcal{E}, \mathcal{F}$, and $P$ are, respectively the energy density, flux, and pressure of the diffuse radiation field, and $\mu_{g}$ was a geometric quantity in our problem (the cosine of the half-angle subtended by the stellar photosphere), but could be treated as an adjustable closure factor in more general situations. For example, the choice $\mu_{g}=1$ would automatically effect a smooth transition from the relation $\varepsilon=3 P$ correct for isotropic radiation under optically thick conditions (when $\mathcal{F} / c \ll \varepsilon$ ) to the relation $\mathcal{E}=P$ appropriate to monodirectional radiation (e.g., optically thin transport at large radial distances from a central source when $\mathcal{F}=c \mathcal{E}$ ). Iterations to determine the correct closure function $\mu_{g}$ should be computationally competitive with the variable Eddington-factor method [83].

Once the flow has been followed through the opacity gap, the matter enters the protostar via an accretion shock. The quasi-hydrostatic evolution of the protostar can be treated as a conventional problem in stellar interiors theory, with the modification of a nonconstant mass and nonstandard surface boundary conditions provided by a radiative accretion shock [22]. Much of the controversy in finite-difference treatments of the evolution of low-mass protostars arose because of inaccuracies in the numerical treatment of this strong radiative shock; thus, a critical part of our analysis was the derivation of total jump conditions that spanned the entire radiative relaxation layer (from upstream point 1 to the viscously relaxed downstream point 2 to the radiatively relaxed downstream point 
3). The derivation rests on the crucial observation that for strong protostellar shocks, the emissive relaxation of viscously shocked gas to the equilibrium radiation temperature must be made in a space which occupies much less than one photon mean free path. The resulting surface boundary conditions for the stellar structure calculation were then the separate conservation of momenta in the matter and radiation fields:

$$
P_{3}+\rho_{3} u_{3}^{2}=P_{1}+\rho_{1} u_{1}^{2} ; \quad P_{\mathrm{rad} 3}=P_{\mathrm{rad} 1},
$$

and the downstream equilibrium temperature for matter and radiation:

$$
T_{3}=\left[\frac{1}{\sigma}\left(\frac{3}{4} F_{\mathrm{rad} 1}-\frac{1}{4} F_{\mathrm{rad} 3}\right)+T_{d}^{4}\right]^{1 / 4},
$$

if the upstream conditions are thin to the propagation of optical photons, and

$$
T_{3}=T_{1},
$$

if the upstream conditions are optically thick. In addition to the above jump conditions, there are the usual ones expressing the conservation of mass and total energy.

Given the above surface boundary conditions, we could follow the evolution of protostars undergoing spherical accretion with high numerical precision [23]; calculations were performed under the assumption that $\dot{M}=10^{-5} M_{\odot} \mathrm{y}^{-1}$, which at the time seemed reasonable since the turbulent and magnetic contribution to an effective value of $a$ were unknown. The results showed that the protostar accumulated mattcr processed through an accretion shock of ever increasing specific entropy so the star remained radiative until deuterium ignited near the center when the stellar mass was about $0.3 M_{\odot}$. A convection zone then spread outward through the star until it became almost entirely convective at a mass of about 0.5 $M_{\odot}$. Apart from this event, nothing dramatic happened to distinguish a particular mass scale for the accreting protostar, and in the actual calculations, the infall was artificially shut off after $10^{5} \mathrm{y}$ when the star had accumulated $1 M_{\odot}$. The surface of the star, which had been kept abnormally hot and luminous by a standing shock then cooled in less than a day and joined a convective (Hayashi) pre-mainsequence track. The disappearance of the infall region would first make the star optically visbile at this point. If the loci of such points in the Hertzsprung-Russell diagram for different shut-off times (i.e., masses) are joined, we have a "birthline" for pre-main-sequence stars of low mass; the birthline corresponding to spherical mass accretion at the rate $\dot{M}=10^{-5} M_{\odot} \mathrm{y}^{-1}$ gave a remarkable fit to the upper envelope for T Tauri stars in Taurus-Auriga, Orion, NGC 7000/1C 5070, and Ophiuchus [84].

Unfortunately, the good agreement between the theoretical birthline and the observed one is marred by three doubts concerning the strict applicability of the 
actual computations to realistic circumstances. First, it is unlikely that the infall would be purely spherical since even a small amount of initial rotation in the oiginal molecular cloud core would have produced accretion partially through a disk. Second, if we adopt Myers's observed value of $a=0.2 \mathrm{~km} \mathrm{~s}^{-1}$, the indicated infall rate from equation (12) is $\dot{M}=2 \times 10^{-6} M_{\odot} \mathrm{y}^{-1}$, not the $1 \times 10^{-5} M_{\odot}$ $y^{-1}$ assumed in the actual calculations. And third, in a spherical treatment, there is no natural justification for shutting off the accretion at the particular masses chosen to give low-mass stars.

The first of the above objections has been given strongest voice by MERCERSMITH, CAMERON, and EPSTEIN [85] who compute the properties of stars that they envisage to be born from disk accretion. In their formalism, the "disk" enters in actual practice only through two postulates: by supplying a rate of mass addition $\dot{M}$ which differs in functional form from equation (12), and by modifying the surface boundary conditions, equations (14) and (15), for a stellar evolution caculation carried out by a spherical code,

$$
\begin{gathered}
4 \pi R_{*}^{2} \sigma T_{g}^{4}=L_{\mathrm{int}}+\xi \frac{G M \dot{M}}{R_{*}}, \\
s(M+\Delta M, t+\Delta t)=\mathbf{s}(M, t),
\end{gathered}
$$

where $L_{\text {int }}$ is the interior luminosity of the star, $T_{g}$ is the temperature of the gas photosphere, $s$ is the specific entropy, $\Delta M \equiv \dot{M} \Delta t$ is the mass added in time increment $\Delta t$, and $\xi$ is a free parameter having a value $\leq 0.5$. Although MERCERSMITH et al. chose to emphasize the differences, in fact, their computed models for $\dot{M}=10^{-5} M_{\odot} \mathrm{y}^{-1}$ behaved quite similarly to the ones computed for direct (spherical) infall, the evolutionary tracks even showing a small "hook" in the $\mathrm{H}$ $\mathbf{R}$ diagram when deuterium burning turns on. The similarities are not terribly surprising; as long as stars have radiative interiors, their interior structures are not very sensitive to the surface boundary conditions (which is why "radiative zero" boundary conditions work as well as they do). For stars which accrete on a time scale short in comparison with their Kelvin-Helmholtz time scale, the same statement holds as long as the entropy $s$ of the newly added material is specified. The specification (16b) that the newly added material has the same $s$ as that which already exists in the layer below is a result which holds approximately (but not exactly) from processing through an infall shock and a settling zone (see fig. 7 of [68]); thus, the "disk-accretion" configurations depend critically only on the assumption of an initial entropy, which was fixed by MERCER-SMITH et al. by taking an initial model close to one computed by WINKLER and NEWMAN [86] for spherical infall.

One can give an even more general argument that the stellar outcome for fixed final mass $M$ will not be very sensitive to the issue of direct infall versus disk accretion as long as the (average) mass accumulation rates $\dot{M}$ are comparable. This argument merely notes that independent of whether star formation occurs by direct infall or disk accretion, with or without spherical symmetry, some healthy 
fraction of the gravitational binding energy of the final object $\sim G M^{2} / R_{*}$ must be radiated away at the stellar surface at a rate $L=4 \pi R_{*}^{2} \sigma T_{g}^{4}$ for a time of order $M / \dot{M}$. The range of $T_{g}$ available to gas photospheres is tightly constrained by opacity considerations; therefore, for given $M$ the final stellar radius $R_{*}$ will primarily depend on $\dot{M}$ :

$$
R_{*} \sim\left(\frac{G M}{4 \pi \sigma \bar{T}_{g}^{4}}\right)^{1 / 3} \dot{M}^{1 / 3}
$$

where $\bar{T}_{g}$ is some appropriate time-average of $T_{g}$. Indeed, a scaling law $R_{*} \propto \dot{M}^{1 / 3}$ brings the computed results of STAHLER, SHU, and TAAM into good agreement with those of WINKLER and NEWMAN (see $[7,81]$ ).

From this conservative point of view, at the very least we may say that infall values like $10^{-6}-10^{-5} M_{\odot} \mathrm{y}^{-1}$ are needed to explain the rough observed locations of $\mathrm{T}$ Tauri stars in the Hertzsprung-Russell diagram. The existence of a welldefined upper envelope suggests the possibility of an even stronger constraint, but if the value $\dot{M}=2 \times 10^{-6} M_{\odot} \mathrm{y}^{-1}$ implied by equation (12) for $a=0.2 \mathrm{~km} \mathrm{~s}^{-1}$ is correct, then scaling in accordance with equation (17) would lead to stellar radii which are a factor of $\sim 1.7$ too small, and therefore "birthline" luminosities which are a factor $\sim 3$ too low. In the next section, we shall present evidence that $\dot{M}=$ $2 \times 10^{-6} M_{\odot} y^{-1}$ is probably the correct choice, at least in Taurus, and therefore, observations are in slight conflict with the model of purely spherically symmetric infall. Given the ample observational evidence concerning the importance of rotation in the formation of binary stars and planetary systems, this should hardly come as a surprise; instead, we should be grateful that the simple spherical theory works as well as it does. Its biggest failing is that it does not give an account of why the infall should stop when it does. Disk accretion per se does no better in this regard, for as long as infall is sustained, matter continues to accumulate either in the star or in the disk.

\section{The Infrared Signature of Protostars}

To test the fundamental notion that stars do form from a process of infall from a molecular cloud core, it is desirable to compute the expected emergent spectral energy distributions of protostars. Numerous groups have performed such calculations for the spherically symmetric problem [87-90], and ADAMS and SHU [91,92] have discussed a fast approximate technique applicable to multi-dimensional situations as well. The fundamental idea is to split the total radiation field $I_{\nu}$ into a directed part which comes from the central source $I_{\nu}^{c}$ (star plus disk) and a diffuse part $I_{\nu}$ which comes from the dust envelope:

$$
I_{\nu}=I_{\nu}^{c}+I_{\nu}
$$

Since the net rate of conversion of radiant energy into other forms (e.g., dissociation of hydrogen molecules, destruction of dust grains) by the infalling matter is small 
in comparison to the total luminosity, and since grain scattering cross-sections ( $\alpha$ $\left.\nu^{4}\right)$ are negligible in comparison with absorption cross-sections $\left(\propto \nu\right.$ or $\left.\nu^{2}\right)$ in the infrared, the specific intensity $I_{\nu}$ of the diffuse photons at positon $\mathbf{r}$ traveling in direction $\mathbf{n}$ is governed by the following time-independent equation of radiative transfer:

$$
\mathrm{n} \cdot \nabla I_{\nu}=\rho \kappa_{\nu}\left[B_{\nu}(T)-I_{\nu}\right]
$$

where $B_{\nu}(T)$ is the Planck function associated with dust grains at temperature $T$, and $\kappa_{\nu}$ is the opacity of the dust. Equation (19) has a formal solution which can be obtained by ray tracing:

$$
I_{\nu}(\mathbf{r}, \mathbf{n})=\int_{0}^{\infty} \kappa_{\nu} \rho\left(\mathbf{r}^{\prime}\right) B_{\nu}\left[T\left(\mathbf{r}^{\prime}\right)\right] \exp \left[-\tau_{\nu}(\mathbf{r}, \mathbf{n}, \boldsymbol{s})\right] d s
$$

where $s$ is the distance traveled by a ray from the source point $\mathbf{r}^{\prime}$ to the field point $\mathbf{r}$,

$$
\text { sn }=\mathbf{r}-\mathbf{r}^{\prime},
$$

and $\tau_{\nu}$ is the optical depth associated with this ray path:

$$
\tau_{\nu}(\mathbf{r}, \mathbf{n}, \boldsymbol{s}) \equiv \int_{0}^{s} \kappa_{\nu} \rho\left(\mathbf{r}^{\prime \prime}\right) d s^{\prime}
$$

with $\mathbf{r}^{\prime \prime} \equiv \mathbf{r}-\boldsymbol{s}^{\prime} \mathbf{n}$

To make use of the formal solution (20) we need calculations of $\kappa_{\nu}, \rho$, and $T$. DRAINE and LEE [93] have recently made comprehensive calculations of the dust opacity $\kappa_{\nu}$. For low-mass protostars, the density distribution $\rho$ can be found by a separate dynamical calculation (see, e.g., [25]). Consistent with this decoupling approximation, the temperature $T$ is to be determined by the selfconsistent requirement that dust grains radiate as much (integral) energy as they absorb:

$$
4 \pi \int_{0}^{\infty} \kappa_{\nu} B_{\nu}(T) d \nu=\int_{0}^{\infty} d \nu \kappa_{\nu} \oint I_{\nu} d \omega
$$

where the last integration is performed over all solid angles of photon propagation and $I_{\nu}$ is given by equation (18), with $I_{\nu}^{c}$ being extincted in the usual fashion along every ray path. Efficient (approximate) techniques based on solutions of the moment equations with heuristic choices for the closure relation (13) can be developed to calculate $T$.

Provided the parameters of the low-mass protostellar models are chosen appropriately, synthetic spectral energy distributions can be constructed which yield good agreement with observations of a class of low-luminosity infrared sources, those which have steep spectra and are found near the centers of dense molecular cloud cores [94]. The mass accretion rates needed to account for the overall luminosity and spectral shape are consistent with $\dot{M}=2 \times 10^{-6} M_{\odot} \mathrm{y}^{-1}$, but not with $\dot{M}=1 \times 10^{-5} M_{\odot} \mathrm{y}^{-1}$. Moreover, to obtain the observed levels of emission shortward of $\lambda=10 \mu \mathrm{m}$, we found it often necessary to adopt initial rates 
of angular rotation for the molecular cloud core of $\Omega=1 \times 10^{-14} \mathrm{~s}^{-1}$ or larger. Such rates are compatible with measured values, lending support to the whole theoretical development.

The infrared signature of protostars is a large hump of radiation peaking at $\sim 10^{2} \mu \mathrm{m}$ with a steep fall-off at shorter wavelengths. The rapid drop of intensity at mid- and near-infrared wavelengths is presumably what caused early groundbased surveys at such bands to miss these sources [95]. Perhaps observers were misled by the predictions of relatively high effective temperatures for the dust photosphere into thinking that there should be more radiation at mid- and nearinfrared wavelengths. For the theoretical infall models, however, it turns out that the color temperatures where the emission peaks are considerably cooler than the effective temperatures of the dust photospheres (defined by where $\sigma T^{4}$ equals the local radiative flux); this characteristic of extended "atmospheres" [83], plus the steep dependence of opacity with decreasing wavelength and the low luminosities of most of the good candidates, probably accounts in large part for the failure of previous searches for true protostars.

Both the overall luminosity and the drop in infrared emission at the shorter wavelengths are exaggerated in the spherical models compared to the real objects. Nonspherical models, which include the radiation from accreting nebular disks that arise when the effects of rotation are included, give better results in these two regards: the luminosity can be lower for a given infall rate because some of the gravitational binding energy released can be stored as kinetic energy of (differential) rotation, and the disk's copious emission in the mid- and near-infrared can supplement the reprocessing of stellar optical photons by the dust envelope to radiation at these wavelengths.

The rotating infall models even give acceptable fits for some infrared sources which are known bipolar outflow sources. This finding supports the idea that well-collimated sources represent objects in which inflow and outflow are taking place simultaneously, indeed, that they represent exactly the transitional phase of evolution between a purely accreting protostar and a fully revealed pre-mainsequence star $[96,97]$. Sketched below is an outline of how rotation might play the crucial role for energizing and collimating such a stellar wind. The accompanying conjecture of how the inflow is eventually reversed closes the logical loop concerning the physical processes which ultimately define the mass of the central star.

\section{Winds, Jets, Bipolar Flows, and the Masses of Formed Stars}

Although no fully self-consistent account of the formation of a rotating protostar by a combination of direct infall and disk accretion is yet available, it is easy to predict some general features. First, as long as the star's interior remains radiative, it is likely to rotate differentially. (The incoming material can hardly know in advance to have a distribution of angular momentum which corresponds to uniform rotation when it is incorporated inside a star.) The store of energy represented by the differential rotation will be a healthy fraction of the total gravitational binding 
energy of the star, which, in turn, is about $10^{3}$ larger than the kinetic energy needed to drive CO bipolar outflows [98]. Thus, if the rotational energy of the star can be converted into outflow with reasonable efficiency and on a reasonable time scale (comparable to or shorter than $M / \dot{M}$ from accretion), it can amply supply the observed wind requirements. However, as long as the star has no internal circulation, there is no obvious mechanism to tap the energy contained in differential rotation. But once fluid does begin to circulate across shear layers, dynamo mechanisms should operate that amplify magnetic fields [99], which can eventually pump energy into driving a stellar wind and coincidentally account for the high levels of surface activity that characterize the atmospheres of the T Tauri stars to be revealed at a later stage.

As long as low-angular momentum material falls in first (see [25]), the protostar is automatically built up with a mechanically stable stratification - a distribution of specific angular momentum which increases outward. Any internal circulation which arises, therefore, has to be driven thermally. Eddington-Sweet circulation is too slow in low-mass protostars where the accretion time is short in comparison with the Kelvin-Helmholtz time, although it might be important in high-mass protostars. Thus, the thermal driving must probably be convection. In the spherical models, convection does not occur in a major way until the onset of deuterium burning. If the same statement applies to models which incoporate a combination of direct infall and disk accretion, then we would have a fundamental understanding of why stars usually form with a mass scale which is roughly marginal for thermonuclear fusion, namely, they keep on accreting mass until their interior temperatures $\left(\propto M_{*} / R_{*}\right)$ rise enough to burn at least the easily fused of all nuclear species, deuterium. And for an infall rate of $2 \times 10^{-6} M_{\odot} y^{-1}$, this will occur typically when the star has a few tenths of a solar mass. In this picture, once $\dot{M} \approx a^{3} / G$ is given, it is stars themselves which become aware of the fundamental combination $\left(\hbar c / G m_{\mathrm{H}}^{2}\right)^{3 / 2} m_{\mathrm{H}}$, not the interstellar medium. To be sure, the quantity which probably matters is the mass addition rate onto the star, $\dot{M}_{*}$, not the total infall rate, $\dot{M}$, onto star plus disk; thus, the exact mass that a star ends up with should also depend on $\Omega$, the pre-collapse rotation rate of the molecular cloud core. In guessing that a few tenths of a solar mass will be typical, we are supposing that disk accretion is efficient and, therefore, $\dot{M}_{*}$ will be rate-limited to $\dot{M}$. If the disk does not always empty nearly all of the mass added to it onto the central star, then it might become sufficiently massive to become gravitationally unstable. Dynamical fragmentation into a multiple star system then becomes a realistic possibility. In all cases, low-mass stars are the likely outcome because, even if nothing else drives the star almost completely convective, deuterium burning will when $\dot{M}_{*} \leq \dot{M}$ is small. In this picture, high mass stars arise only if $\dot{M}$ can be made large, either because an unstable cloud core had an initially high temperature or because it suffered implosion by external means.

How would breakout of the stellar wind in a low-mass protostar occur in practice? When deuterium ignites (slightly off-center in the spherical models), buoyant 
motions across shearing layers would probably try to bring the material in the convection zone to a state of more nearly uniform rotation, releasing energy in the process. Some part of this energy will go into amplifying seed magnetic fields; another part, to overturning the stable entropy gradient and driving the outer boundary of the convection zone upwards. However, as long as there is still a substantial outer radiative envelope, the heat released (and the magnetic fields generated) cannot make its way to the surface, and the radius of the star will expand. Perhaps it is this expansion which reconciles the observed "birthline" for

T Tauri stars with the relatively low mass-accretion rate of $\sim 2 \times 10^{-6} M_{\odot} \mathrm{y}^{-1}$. In any case, when the outer radiative zone becomes thin enough, thermal equilibration of magnetic flux tubes [100] will occur rapidly to enable them to buoy out to the radius of the standing infall shock. There they will pile up, like steam in a pressure cooker with the lid on, until the magnetized plasma builds up enough head to break out.

Just as in a pressure cooker, however, breakout will not immediately blow off the entire lid (the ram pressure of the inflow); it will occur first through the channel of least resistance (the safety valve). One glance at figure 6e of [25] should make clear that the weakest point occurs at the rotational pole(s) of the accreting protostar. Thus, the gases of the incipient stellar corona should initially be channeled up and out of the rotational poles of the system, forming two highly collimated stellar jets. The heads of the jet will plow through the infalling cloud envelope, eventually sweeping up enough material to account for the $\mathrm{CO}$ lobes of the observed bipolar outflows. The prediction is, therefore, that highly collimated outflow sources should generally correspond to deeply embedded objects, in which outflow and inflow are occurring simultaneously. This prediction is in accord with the finding that bipolar outflow sources often have steep spectral energy distributions in the infrared which make them virtually indistinguishable from (protostellar) candidates for pure infall.

As time proceeds, more and more of the rotating inflowing matter falls preferentially on the disk rather than on the star. The "lid" of the pressure cooker will weaken relative to the "steam," and the stellar jet will widen, eventually to sweep outwards over all $4 \pi$ steradians and bring an end to the infall phase. An outside observer will then be able to see optically a $\mathrm{T}$ Tauri star, newly born on a convective (Hayashi) pre-main-sequence track, still bubbling with residual surface activity [101-103], and surrounded perhaps by a remnant nebular disk. The slow rotation rates observed for $\mathrm{T}$ Tauri stars [104], which came as a major surprise when first discovered, may now be naturally attributed to the large magnetic braking associated with an earlier period of intense mass loss. In this fashion will a solar-type star dramatically make its existence known to creatures with eyes sensitive only to visible radiation.

\section{References}

1. Hayashi, C. 1966, Ann. Rev. Astr. Ap., 4, 171. 
2. Larson, R. B. 1969, M. N. R. A.S., 145, 271.

3. Narita, S., Nakano, T., and Hayashi, C. 1970, Prog Theor. Phys., 43, 942.

4. Hayashi C. 1970, in Evolution Stellaire avant la Sequence Principale, 16me Liege Symposium (University de Liege), p. 127.

5. Appenzeller, I., and Tscharnuter, W. 1975, Astr. Ap., 40, 397.

6. Westbrook, C.K., and Tarter, C. B. 1975, Ap. J., 200, 48.

7. Winkler, K. H., and Newman, M. J. 1980, Ap. J., 236, 201.

8. Larson, R. B. 1978, M. N. R. A. S., 184, 69.

9. Narita, S., and Nagazawa, K. 1978, Prog. Theor. Phys., 59, 1018.

10. Norman, M. L., and Wilson, J. R. 1978, Ap. J., 224, 497.

11. Boss, A. P. 1980, Ap. J, 236, 619.

12. Scott, E. H., and Black, D. C. 1980, Ap. J., 239, 166.

13. Tohline, J. E. 1980, Ap. J., 236, 160.

14. Bodenheimer, P., Tohline, J. E., and Black 1980, Ap. J., 242, 209.

15. Rozyczka, M., Tscharnuter, W. M., Winkler, K. H., and Yorke, H. W. 1980 , Astr. Ap., 83, 118.

16. Black, D. C., and Scott, E. H. 1982, Ap. J., 263, 696.

17. Boss, A. P. 1984, Ap. J., 277, 768.

18. Penston, M. V. 1969, M. N. R. A. S., 144, 425.

19. Larson, R. B. 1969, M. N. R. A. S., 145, 271.

20. Shu, F. H. 1977, Ap. J., 214, 488.

21. Hunter, C. 1977, Ap. J., 218, 834.

22. Stahler, S. W., Shu, F. H., and Taam, R. E. 1980, Ap. J., $241,637$.

23. Stahler, S. W., Shu, F. H., and Taam, R. E. 1980, Ap. J., 242, 226.

24. Stahler, S. W., Shu, F. H., and Taam, R. E. 1981, Ap. J., 248, 727.

25. Terebey, S., Shu, F. H., and Cassen, P. 1984, Ap. J., 286, 529.

26. Larson, R. B. 1973, Ann. Rev. Astr. Ap., 11, 219.

27. Bodenheimer, P., and Black, D. C. 1978, in Protostars and Planets, ed. T. Gehrels (Tucson: University of Arizona Press), p. 288.

28. Bodenheimer, P. 1980, in IAU Symposium No. 93, Fundamental Problems in the Theory of Stellar Evolution, ed. D. Sugimoto, D. Q. Lamb, and D. N. Schramm (Dordrecht: Reidel), p. 5. 
29. Tscharnuter, W. M. 1980, Space Sci. Rev., 27, 235.

30. Tohline, J. E., Fund. Cosmic Phys., 8, 1.

31. Iben, I., and Renzini, A. 1983, Ann. Rev. Astr. Ap., 21, 271.

32. Miller, G. E., and Scalo, J. M. 1979, Ap. J. Suppl., 41, 513.

33. Hoyle, F. 1953, Ap. J., 118, 513.

34. Hunter, C. 1964, Ap. J., 139, 570.

35. Low, C., and Lynden-Bell, D. 1976, M. N. R. A. S., 142, 1431.

36. Boss, A. P. 1981, Ap. J., $250,636$.

37. Silk, J. 1982, Ap. J., 256, 514.

38. Larson, R. B. 1985, M. N. R. A. S., 214, 379.

39. Shu, F. H. 1985, in IAU Symposium No. 106, The Milky Way, ed. H. van Woerden, R. J. Allen, and W. B. Burton (Dordrecht: Reidel), p. 561.

40. Schwartz, R. D. 1983, Ann. Rev. Astr. Ap., $21,209$.

41. Cohen, M. 1984, Physics Reports, 116, no. 4, 173.

42. Lada, C. J. 1985, Ann. Rev. Astr. Ap., 23, 267.

43. Welch, W. J., Vogel, S. N., Plambeck, R. L., Wright, M. C. H., and Biegieg, J. H. 1985, Science, in press.

44. Sargent, A. I. 1977, Ap. J., 218, 736.

45. Blitz, L., and Thaddeus, P. 1980, Ap. J., 241, 676.

46. Zuckerman, B., and Palmer, P. 1974, Ann. Rev. Astr. Ap., 12, 279.

47. Field, G. B. 1978, in Protostars and Planets, ed. T. Gehrels (Tucson: University of Arizona Press), p. 243.

48. Norman, C., and Silk, J. 1980, Ap. J., 238, 158.

49. Larson, R. B. 1981, M. N. R. A. S., 194, 809.

50. Mestel, L. 1965, Quart. J. R. A. S., 6, 161.

51. Mouschovias, T. Ch. 1976, Ap. J., 207, 141.

52. Goldsmith, P. F., and Arquilla, R. 1985, in Protostars and Planets II, ed. D.

C. Black and M. S. Matthews (Tucson: University of Arizona Press), in press.

53. Spitzer, L. 1968, Diffuse Matter in Space (New York: Intcrscience).

54. Mouschovias, T. Ch., and Spitzer, L. 1976, Ap. J., 210, :26.

55. Troland, T. H., and Heiles, C. H. 1985, Ap. J., in press.

56. Vrba, F. J., Strom, K. M., and Strom, S. E. 1976, A. J., 81, 958. 
57. Arons, J., and Max, C. E. 1975, Ap. J. Letters, 196, L77.

58. Mestel, L. 1985, in Protostars and Planets II, ed. D. C. Black and M. S. Matthews (Tucson: University of Arizona Press), in press.

59. Cohen, L. V., and Kuhi, L. V. 1979, Ap. J. Suppl., 41, 743.

60. Myers, P. C., and Benson, P. J. 1983, Ap. J., 266, 309.

61. Vrba, F. J., Strom, K. M., Strom, S. E., and Grasdalen, G. L. 1975, Ap. J., 197, 77.

62. Lada, C. J., and Wilking, B. A. 1984, Ap. J., 287, 610.

63. Mestel, L., and Spitzer, L. 1956, M. N. R. A. S., 116, 503.

64. Nakano, T., and Tademaru, E. 1972, Ap. J., $173,87$.

65. Mouschovias, T. Ch. 1981, in IAU Symposium No. 91, Fundamental Problems in the Theory of Star Formation, ed. D. Sugimoto, D. Q. Lamb, and D. N. Schramm (Dordrecht: Reidel), p. 27.

66. Nakano, T. 1982, Pub. Astr. Soc. Japan, 34, 337.

67. Mouschovias, T. Ch., Paleologou, E. V., and Fiedler, R. A. 1985, Ap. J., 291, 772.

68. Draine, B. T., Roberge, W. G., and Dalgarno, A. 1983, Ap. J., 264, 485.

69. Shu, F. H. 1983, Ap. J., 273, 202.

70. Lizano, S., and Shu, F. H. 1986, in preparation.

71. Gillis, J., Mestel, L., and Paris, R. B. 1974, Ap. Space Sci., 27, 167.

72. Gillis, J., Mestel, L., and Paris, R. B. 1979, M. N. R. A. S., 187, 311.

73. Mouschovias, T. Ch., and Paleologou, E. V. 1979, Ap. J., 230, 204.

74. Mouschovias, T. Ch., and Paleologou, E. V. 1980, Ap. J., 237, 877.

75. Bodenheimer, P., and Sweigart, A. 1968, Ap. J., 152, 515.

76. Whitworth, A., and Summers, D. 1985, M. N. R. A. S., 214, 1.

77. Nakano, T. 1981, Prog. Theor. Phys. Suppl. No. 70, 54.

78. Elmegreen, B. G. 1979, Ap. J., 232, 729.

79. Nakano, T., and Umebayashi, T. 1980, Pub. Astr. Soc. Japan, 32, 613.

80. Myers, P. C. 1985, in Protostars and Planets $I I$, ed. D. C. Black and M. S. Matthews (Tucson: University of Arizona Press), in press.

81. Ho, P. T. P., and Townes, C. H. 1983, Ann. Rev. Astr. Ap., 21, 239.

82. Hayashi, C., Hoshi, R., and Sugimoto, D. 1962, Prog. Theor. Phys. Suppl. No. 22. 
83. Mihalas, D. 1978, Stellar Atmospheres (San Francisco: Freeman).

84. Stahler, S. W. 1983, Ap. J., 274, 822.

85. Mercer-Smith, J. A., Cameron, A. G. W., and Epstein, R. I. 1984, Ap. J., $287,445$.

86. Winkler, K. H., and Newman, M. J. 1980, Ap. J., 238, 311.

87. Larson, R. B. 1969, M. N. R. A. S., 145, 297.

88. Yorke, H. W., and Krugel, E. 1977, Astr. Ap., 54, 183.

89. Bertout, C., and Yorke, H. W. 1978, in Protostars and Planets, ed. T. Gehrels (Tucson: University of Arizona Press), p. 648.

90. Yorke, H. W., and Shustov, B. M. 1981, Astr. Ap., 98, 125.

91. Adams, F. C., and Shu, F. H. 1985, Ap. J., in press.

92. Adams, F. C., and Shu, F. H. 1986, Ap. J., in preparation.

93. Draine, B. T., and Lee, H. M. 1984, Ap. J., 285, 89.

94. Myers, P. C., Mathieu, R. D., Benson, P. J., Beichman, C., and Fuller, G. A. 1986 , in preparation.

95. Wynn-Williams, C. G. 1982, Ann. Rev. Astr. Ap., 20, 587.

96. Shu, F. H., and Terebey, S. 1984, in Cool Stars, Stellar Systems, and the Sun, ed. S. Baliunas and L. Hartmann (Berlin: Springer-Verlag), p. 78.

97. Cassen, P., Shu, F. H., and Terebey, S. 1985, in Protostars and Planets II, ed. D. C. Black and M. S. Matthews (Tucson: University of Arizona Press), in press.

98. Bally, J., and Lada, C. J. 1983, Ap. J., 265, 824.

99. Parker, E. N. 1979, Cosmical Magnetic Fields (Oxford University Press).

100. Parker, E. N. 1984, Ap. J., 286, 666.

101. Herbig, G. 1962, Adv. Astr. Ap. 1, 47.

102. Kuhi, L. V. 1964, Ap. J., 140, 409.

103. Calvet, N., Basri, G., and Kuhi, L. V. 1984, Ap. J., 277, 725.

104. Vogel, S., and Kuhi, L. 1981, Ap. J., 245, 960.

\section{Discussion}

M. L. Norman: You stated that turbulence was unimportant in supporting the dense cores in large molecular clouds. Could you comment on the impressive correlation Richard Larson has found between radio line widths and core compactness, which Larson interprets as virial equilibrium between gravity and turbulent pressure? 
F. Shu: I only said that "turbulence" could not be wholly dominant over magnetic fields, not that it was unimportant. As far as the correlation found by Larson goes, let me make two comments. First, the exponent in the power law is now believed by the observers to be different from that appropriate for a classical Kolmogorov spectrum (arising from a cascade of eddies). Thus, the "turbulence" is probably not eddylike. The fact that it is still a power law is not surprising - this would probably arise whenever there is a large dynamic range between the scale at which energy is fed into the disturbances (waves?), and the scale at which it is dissipated. Second, the interpretation of virial equilibrium is logically disconnected from the nature of the "turbulence." Moreover, it is contaminated by selection effects which tend to pick up certain column densities when observing in certain molecules. Thus, although $I$ do not object to the vague idea of a kind of "turbulent virial equilibrium," I'd prefer to see the concept made more definite before I'd accept its importance in any truly fundamental sense. My position is, in any case, that such means of support cannot be wholly dominant over magnetic effects, and that both are doomed to yield eventually to self-gravity in the cores of molecular clouds.

V. Icke: It is not surprising that the turbulent spectrum is not exactly Kolmogorov since (a) the turbulence here is compressible, and (b) it is not isotropic because of the importance of galactic dynamics on this scale.

F. Shu: (a) I agree the gas is compressible, but I don't think the velocities are associated with eddies for the reason that I mentioned in my talk - because otherwise the magnetic feld would get all tangled up and show no large-scale order. If chaotic velocities are present, they probably involve wavelike motions rather than eddylike ones. (b) On the scales important to star formation ( $\sim 10^{17}$ $\mathrm{cm}$ ), the gas can hardly know that it lives in a galaxy.

M. Shull: If you consider globular clusters as an ideal example of your collapse calculations, what predictions would you make concerning the synchronous collapse of old stars? For example, does it follow that many stars must form by secondary (triggered) collapse?

F. Shu: If the idea of core formation by ambipolar diffusion is correct, then present-day star formation in $\mathrm{T}$ associations is "unsynchronized" (time scale $10^{7} \mathrm{y} \gg$ the $10^{5}$ or $10^{6} \mathrm{y}$ required to form a star once collapse ensues) because the ionization fraction is maintained by cosmic rays at a certain level. For lower ionization fractions (or no magnetic fields), the synchronization would be much better because magnetic fields will be less able to delay collapse. Also, in the absence of coolants (metals and grains), the accretion rate $\dot{M}$ of forming stars would be much larger because the temperatures will be higher. Star formation in such circumstances will be quite different from those in present-day GMCs.

R. Mundt: You suggested that we can expect well-collimated jets only from the youngest stars (observable as IR sources only and not as visible objects), for which 
the infalling matter is so dense that it provides sufficient kinetic pressure to confine the flow. This is only correct if the driving force of the flow is not decreasing with time (the pressure of your "pressure cooker"). We can't expect that since the infalling matter may drive the outflow.

F. Shu: That's a good point.

R. Opher: How do you solve the angular momentum problem in your models?

F. Shu: It's solved, as far as collapse to binary-star or solar-system dimensions are concerned, by magnetic braking of the cloud cores to rotation rates typical of their envelopes. It's not solved for the rapidly rotating protostar until after it begins to turn on a strongly magnetized wind; indeed, it's the excess store of energy in differential rotation which provides the basic source for driving the wind.

R. Opher: If magnetic fields are the primary mechanism preventing collapse in molecular clouds, shouldn't we observe highly flattened objects?

F. Shu: Along the direction of the field other means of support, e.g., rotation, or probably more importantly, "turbulence" in the form of many Alfven waves, will be needed. My point was simply that observations suggest that these other means cannot be wholly dominant over the magnetic fields.

R. Opher: To form a star, we are dealing with densities $>10^{22} \mathrm{~cm}^{-3}$. Already at $\sim 10^{12} \mathrm{~cm}^{-3}$, however, the gas is opaque and the isothermal assumption breaks down. Have you included this effect in your calculations?

F. Shu: Yes.

L. Anderson: Since the original cloud angular momentum also does not know of Chandrasekhar's number of $\mathrm{H}$ atoms required to ignite a star, how does rotation limit the accretion?

F. Shu: To tap the store of energy represented by the differential rotation of the star requires the onset of convection, which then amplifies magnetic fields by dynamo activity, leading to a wind. Thus, to turn on the wind in a low-mass star requires it to have at least the mass to burn deuterium (with the radius that comes out of the self-consistent protostellar accretion problem).

L. Anderson: A massive unstable disk will change the "pressure cooker" model and jet confinement - a possible observational difference.

F. Shu: Yes, for example, if the massive disk forms another star, the resultant perturbation on the jet from the other star could make it become wiggly.

R. Blandford: How do you make binaries? 
F. Shu: Clearly, not by imposing axial symmetry as we have done in our calculations to date. For forming close binaries, this could be done by developing a gravitational instability in an axisymmetric disk which becomes sufficiently massive (comparable to the central star) if (viscous) transport in the disk fails to keep up with the addition of matter to it by infall. For wide binaries, this method probably fails because it is difficult to imagine how the other star could manage to sweep up all the matter in a large disk. Here, it's probably necessary to invoke two centers of accretion from the outset.

S. Owocki: (a) You said magnetic fields would be important in preventing freefall colllapse. Where are the magnetic fields in your series of collapse snapshots? (b) What drives the wind? (c) Why doesn't infall occur over the pole? (d) What observational characteristics make it possible to infer $30 \mu \mathrm{G}$ magnetic fields in your problem, whereas Mundt told us this morning that $B<10^{4} \mathrm{G}$ would not be observable in protostars?

F. Shu: (a) Magnetic fields were invoked only to select a preference for initially uniformly rotating states. They were left out for the dynamical part of the calculation by Terebey, Shu, and Cassen. We're contemplating putting them back in a pertubational sense similar to how we incorporated rotation. (b) The wind is driven by a combination of magnetic effects (Alfven waves?) and hot gas produced by the activity associated with convection in a differeptially rotating protostar of low mass. This, at least, is the speculation, but honest (or half-honest!) calculations are needed. (c) It does; it's just weaker there at late times than elsewhere (because most of the low angular momentum material has already fallen in). (d) The line widths are much narrower in the interstellar medium than in jets. 